\title{
Numerical analysis of oil-water two-phase flow in pipe systems
}

\section{Introduction}

Crude oil sources are remote nowadays from most of processing centres and refineries. The main problem of the conventional and heavy oil transportation in long horizontal pipes is that it has high pressure losses because of its high viscosity. The easiest way to reduce the pressure losses is to mix it with water. However, the multiphase oilwater pipe flow problem is very complicated. It is highly dependent on many parameters such as the phases input fraction, mixture velocity, pipe diameter, temperature and pressure. The frictional pressure drop of oil-water mixture increases much stronger in a pipeline as compared to oil mass flow rate increase if the velocity is simply increased. Large difference in the viscosity between oil and water makes the study of flow patterns much more complicated. Mathematical modelling and numerical analysis of oil-water two-phase pipe flows may improve our knowledge on the oil-water pipe flow transport mechanisms.

The majority of the experimental research works in oil-water mixture flow in pipes were focused to investigate various aspects of multiphase pipe flows of oil and water that include identification of flow patterns such as the stratified, dispersed, intermittent, core-annular and the combination of them. ${ }^{1-3}$ Flow characteristics and hydrodynamic behaviour on vertical and horizontal pipe flows at different volume fraction of phases ${ }^{4-7}$ were investigated experimentally as well as the effect of the viscosity and density on the fluid flow behaviour. ${ }^{1}$ The high viscosity crude and heavy oil were used in measurements ${ }^{3,8,9}$ also. Experimental investigation of the dual continuous flow phenomena in oil-water mixture flow in horizontal pipes was performed in. ${ }^{5,9} \mathrm{~A}$ distribution of the phases in a pipe cross section was determined. The inversion flow phenomena in a vertical pipe for dispersed oil-water flows were experimentally studied by $\mathrm{Hu}$ \& Angeli. ${ }^{4}$ Pressure reduction was observed in both cases when water was fed into the oil continuous flow and when oil was fed into the water.

Mathematical modelling and numerical analysis such as 3D CFD simulations are becoming more attractive nowadays. They offer high flexibility in studies involving parametric and especially topological changes. Two-fluid Eulerian-Eulerian scheme is the most frequently used method to simulate two-phase oil-water pipe flows where both phases are considered as the continuous and interpenetrating. However, the accuracy in the pressure drop and hold-up prediction is relatively poor in most of studies. ${ }^{9-11}$ Two-fluid scheme of CFD solver ANSYS Fluent was used by Parvini et al. ${ }^{12} \&$ Walvekar et al. ${ }^{13}$ to study various aspects of flow properties. The effect of drag, lift and added mass forces was examined numerically. The lift force was found to be more important than the forces associated with the turbulent dispersion and added mass. ${ }^{12,13}$ An attempt to use CFD model to predict oil-water flow patterns is presented in $^{14}$ using Eulerian-Eulerian approach closed by the standard k-epsilon turbulence model. The drag, lift, and turbulent dispersion forces are considered in the study. The model validation against published experimental data showed reasonable agreement. Cazarez et al. ${ }^{15}$ also developed a one-dimensional thermal transient model that was applied to three-phase heavy oil-water-gas
Volume 2 Issue 3 - 2017

\author{
E Burlutskii \\ Department of petroleum engineering, University of \\ Nottingham, UK
}

Correspondence: $\mathrm{E}$ Burlutskii, Department of petroleum engineering, University of Nottingham, UK, Tel (0) II 57486393 Fax (0) II5 95 । 3667 ,

Email Evgenii.Burlutskii@nottingham.ac.uk

Received: January 02, 2017 | Published: April 19, 2017

bubbly flow in upward vertical pipes. The model was able to predict the pressure, temperature and velocity profiles of the flow. A onedimensional thermal compressible transient model was developed in. ${ }^{16,17}$ It was applied to compressible gas-oil and gas-water flow in pipes. The condensation of liquid oil from gas phase was considered and validated against measurements. In addition, the stratified oilwater flow behaviour were also studied numerically using the volume of fluid (VOF) scheme and the level set method such as those by AlYaari et al. ${ }^{18}$ \& Kaushik et al. ${ }^{19}$ and Gada et al. ${ }^{20}$

\section{Conclusion}

There is, however, very limited work ${ }^{21,22}$ where the Lagrangian approach was used for modelling of dispersed two-phase oil-water droplet flows in pipes. The effect of the shear-lift force on oil droplet movement in water fluid flow is investigated numerically using Eulerian-Lagrangian model in Burlutskiy et al. ${ }^{21}$ The ability of ANSYS Fluent and Open FOAM solvers to simulate turbulent dispersed particle-laden flows is tested in Greifzu et al. ${ }^{22}$ The effect of the dispersion model on the particle motion is investigated in the study. The numerical values predicted using Eulerian-Lagrangian point-particle model for the dispersed phase and Reynolds-averaged Navier-Stokes equations for the fluid phase are validated against published experimental data. The numerical study shows that particle dispersion is slightly under-predicted when ANSYS FLUENT solver is used, whereas the applied solver in Open FOAM overestimates the dispersion somewhat. The Largangain method provides much more advantages in modelling of dispersed two-phase flows as compared to other well-know methods such as mixed, two-fluid, VOF, level set and their modifications. ${ }^{23}$ The Lagrangian approach resolves the motion of droplets in the continuous fluid flow field by taking of all relevant forces into account. This approach considers the droplet phase as a collection of individual discrete particles of certain size represented by centres of spheres. This gives more accurate and complete information about the individual droplet behaviour as compared to the two-fluid scheme where droplets are considered as the continuous phase having averaged properties. Employment of the Lagrangian approach may significantly help in study of the transition criteria from one flow regime to another one, e.g. from fully dispersed to stratified flow regime, especially in the case of two-phase flow in a horizontal pipeline where the gravity acts on the direction which is perpendicular to the mixture flow direction and the slip between phases is observed. 


\section{Acknowledgements}

None.

\section{Conflict of interest}

The author declares no conflict of interest.

\section{References}

1. ME Charles, GW Govier, GW Hodgson. The horizontal pipeline flow of equal density oil-water mixtures. The Canadian Journal of Chemical Engineering. 1961;39(1):27-36.

2. XX Xu. Study of oil-water two phase flow in horizontal pipelines. Journal of Petroleum Science and Engineering. 2007;59:43-58.

3. W Wang, J Gong, P Angeli. Investigation of heavy crude-water two phase flow and related flow characteristics. International Journal of Multiphase Flow. 2011;37:1156-1164.

4. B Hu, P Angeli. Phase inversion and associated phenomena in oil-water vertical pipeline. The Canadian Journal of Chemical Engineering. 2006;84(1):1-14.

5. JY L Lum, TAl Wahaibi, PAngeli. Upward and downward inclination oilwater flows. International Journal of Multiphase Flow. 2006;32(4):413 435 .

6. K Piela, R Delfos, G Ooms, et al. Experimental investigation of phase inversion in an oil-water flow through a horizontal pipe loop. International Journal of Multiphase Flow. 2006;32:1087-1099.

7. K Piela, R Delfos, G Ooms, et al. On the phase inversion process in an oil-water pipe flow. International Journal of Multiphase Flow. 2008;34:665-677.

8. AC Bannwart, OMH Rodriguez, CHM de Carvalho, et al. Flow patterns in heavy crude oil-water flow. Trans ASME. 2004;126(3):184-189.

9. J Lovick, P Angeli. Experimental studies on the dual continuous flow pattern in oil-water flows. International Journal of Multiphase Flow. 2004;30:139-157.

10. N Brauner, D Moalem Maron. Two phase liquid-liquid stratified flow. Physico Chemica. Hydrodynamics. 1989; 11(4):487-506.

11. N Brauner, D Moalem Maron, J Rovinsky. A two-fluid model for stratified flows with curved interfaces. International Journal of Multiphase Flow. 1998;24:975-1004.
12. M Parvini, B Dabir, SA Mohtashami. Numerical simulation of oil dispersions in vertical pipe flow. Journal of Japanese Petroleum Institute. 2010;53(1):42-54.

13. RG Walvekar, SYT Choong, SA Hussain, et al. Numerical study of dispersed oil-water turbulent flow in horizontal tube. Journal of Petroleum Science and Engineering. 2009;65:123-128.

14. H Pouraria, JK Seo, JK Paik. Numerical modelling of two-phase oil--water patterns in a subsea pipeline. Ocean Engineering. 2016;115(15):135148.

15. O Cazarez, D Montoya, AG Vital, et al. Modelling of three-phase heavy oil-water-gas bubbly flow in upward vertical pipes. International Journal of Multiphase Flow. 2010;36:439-448.

16. E Burlutskiy. Numerical analysis of rapid gas decompression from a pipeline in different natural gas mixtures under low temperature conditions and water presence in a pipe International Society of Offshore and Polar Engineers, Rhodes, Greece. 2012;1:1293-1299.

17. E Burlutskiy. Numerical analysis of phase behaviour during rapid decompression of rich natural gases. Process Safety and Environmental Protection. 2014;92(6):555-564.

18. MAAl Yaari, BF Abu Sharkh. CFD prediction of stratified oil-water flow in a horizontal pipe. Asian Transactions on Engineering. 2011;1(5):6875 .

19. VVR Kaushik, S Ghosh, G Das, et al. CFD simulation of core-annular flow through sudden contraction and expansion. Journal of Petroleum Science and Engineering. 2012;86-87:153-164.

20. VH Gada, S Sharma. Analytical and level-set method based numerical study on oil-water smooth/wavy stratified-flow in an inclined planechannel. International Journal of Multiphase Flow. 2012;38:99-117.

21. E Burlutskiy, CK Turangan. A computational fluid dynamics study on oil-in-water dispersion in vertical pipe flows. Chemical Engineering Research and Design. 2015;93:48-54.

22. F Greifzu, C Kratzsch, T Forgber, et al. Assessment of particle-tracking models for dispersed particle-laden flows implemented in OpenFOAM and ANSYS FLUENT. Engineering Applications of Computational Fluid Mechanics. 2016;10(1):30-43.

23. E Bourloutski, AM Bubenchikov, AV Starchenko. The comparison of two approaches to numerical modelling of gas- particles turbulent flow and heat transfer in a pipe. Mechanics Research Communications. 2002;29(5):437-445 